\title{
Local boundedness of variational solutions to evolutionary problems with non-standard growth
}

\section{Thomas Singer}

\begin{abstract}
We prove the local boundedness of variational solutions and parabolic minimizers to evolutionary problems, where the integrand $f$ is convex and satisfies a non-standard $p, q$-growth condition with
\end{abstract}

$$
1<p \leq q \leq p \frac{n+2}{n} .
$$

A function $u: \Omega_{T}:=\Omega \times(0, T) \rightarrow \mathbb{R}$ is called parabolic minimizer if it satisfies the minimality condition

$$
\int_{\Omega_{T}} u \cdot \partial_{t} \varphi+f(x, D u) \mathrm{d} z \leq \int_{\Omega_{T}} f(x, D u+D \varphi) \mathrm{d} z
$$

for every $\varphi \in C_{0}^{\infty}\left(\Omega_{T}\right)$. Moreover, we will show local boundedness for parabolic minimizers, if $f$ satisfies an anisotropic growth condition.

Mathematics Subject Classification. 35A15, 35K86, 49J40, 49N60.

Keywords. Parabolic minimizers, Regularity, Non-standard growth.

\section{Introduction}

We are interested in the regularity of variational solutions, where the integrand $f$ satisfies a non-standard $p, q$-growth condition

$$
\nu|\zeta|^{p} \leq f(x, \zeta) \leq L\left(1+|\zeta|^{q}\right)
$$

for $1<p \leq q \leq p \frac{n+2}{n}$ and $0<\nu \leq L$. The formal corresponding differential equation is

$$
\partial_{t} u-\operatorname{div} \partial_{\zeta} f(x, D u)=0,
$$

but since we do not assume that $f$ is differentiable, the PDE above may have no meaning at all. Moreover, such an equation would only be well-defined, if the weak solution $u$ belongs to the space $L^{q}\left(0, T ; W^{1, q}(\Omega)\right)$, but the theory does not ensure the existence of such weak solutions. To overcome these problems, we consider the notion of variational solutions, which was introduced by 
Lichnewsky and Temam [12] in the context of evolutionary parametric minimal surface equations. In the context of parabolic equations with $p, q$-growth, the notion of variational solutions has been introduced by Bögelein et al. in [1]. Therein they showed existence of variational solutions associated to a convex integrand $f$, only assuming that $f$ fulfils a coercivity condition. In this paper we establish an $L_{\mathrm{loc}}^{\infty}$-bound for these solutions. In order to show this, we will use a parabolic version of the De Giorgi-classes, which was introduced by DiBenedetto in [6]. The analogous elliptic problem is treated in $[8,14,16]$, where the convexity of $f$ and a $\Delta_{2}$-condition is required. For integrands with $p, q$-growth, it is crucial that the gap between $p$ and $q$ is not too large. Otherwise, there exist examples of unbounded solutions (cf. [13]). In [14,16] local boundedness of minimizers to elliptic variational integrands is shown, if

$$
1<p \leq q<p^{\star}=\frac{n p}{n-p}
$$

holds. Here, the embedding $W^{1, p}(\Omega) \hookrightarrow L^{q}(\Omega)$ is compact. In [8], this result is extended to the case $q=p^{\star}$ where the Sobolev embedding is only continuous. However, it is not possible to state an explicit $L^{\infty}$-bound in this case. In this paper, we prove boundedness of parabolic minimizers, provided the gap between $p$ and $q$ is

$$
1<p \leq q \leq p \frac{n+2}{n}=: p_{\star} .
$$

The upper bound $q \leq p_{\star}$ stems from the parabolic embedding. Just as in the elliptic setting, it is not possible to specify an explicit $L^{\infty}$-bound in the limit case $q=p_{\star}$. Furthermore, we only need the convexity of the integrand $f$. This assumption is essential for proving a Caccioppoli inequality, since $f$ satisfies only a non-standard growth condition. In the proof we have to handle the lack of regularity of parabolic minimizers in time, a problem which can, due to the growth condition, not be treated by a time regularization method like Steklov averages. But we will use the methods of [2] to show that $\partial_{t} u \in L^{2}\left(\Omega_{T}\right)$ holds for a variational solution $u$, if it possess time-independent boundary data. Thus we can prove that these solutions are locally bounded.

We also consider parabolic minimizers of functionals, where the integrand $f$ satisfies an anisotropic growth condition of the form

$$
\nu \sum_{i=1}^{n}\left|\zeta_{i}\right|^{p_{i}} \leq f(x, t, u, \zeta) \leq \sum_{i=1}^{n} L\left(1+\left|\zeta_{i}\right|^{p_{i}}\right) .
$$

If we take $p=\min \left\{p_{i}\right\}$ and $q=\max \left\{p_{i}\right\}$, we observe that this is a special case of $p, q$-growth. Here we can additionally allow a $u$-dependency for $f$ and do not need a convexity assumption. This stems from the fact that we have more structure conditions for the integrand $f$. Furthermore, we do not require any information for the boundary data, since we are able to use Steklov averages to compensate the lack of regularity in time. The analogous result for parabolic equations with anisotropic growth conditions has been proved in [15]. Therein the assumption 


$$
p_{i} \leq p_{\star} \quad \text { with } \quad \frac{1}{p}=\frac{1}{n} \sum_{i=1}^{n} \frac{1}{p_{i}}
$$

is needed for the exponents $p_{i}$, which is exactly the same condition we need in this paper. In the elliptic setting, an $L_{\mathrm{loc}}^{\infty}$-bound for minimizers of integrands satisfying an anisotropic or respectively a $p, q$-growth condition is proven in [3], where $p_{i} \leq p^{\star}$ or respectively $p \leq p^{\star}$ is needed. Analogous results for weak solutions of systems are proven in [4]. Of course, the coefficients must satisfy stronger assumptions in order to show regularity. For more details we refer to $[3,5]$ and the references given there.

\subsection{Variational integrands with $p, q$-growth}

Now we formulate our results for variational solutions, where the integrand $f$ satisfies and non-standard $p, q$-growth condition. Therefore, let $\Omega \subset \mathbb{R}^{n}$ be an open bounded domain and $\Omega_{T}:=\Omega \times(0, T)$ describes the space-time cylinder for $T>0$. The integrand $f: \Omega \times \mathbb{R}^{n} \rightarrow \mathbb{R} \cup\{\infty\}$ is supposed to be a Carathéodory-function and to fulfil the following convexity and growth assumptions:

$$
\left\{\begin{array}{l}
\mathbb{R}^{n} \ni \zeta \mapsto f(x, \zeta) \text { is convex for a.e. } x \in \Omega \\
\nu|\zeta|^{p} \leq f(x, \zeta) \leq L\left(|\zeta|^{q}+1\right), \quad \forall(x, \zeta) \in \Omega \times \mathbb{R}^{n},
\end{array}\right.
$$

for some $0<\nu \leq L$. For the initial and boundary datum $u_{0}$ we assume that

$$
u_{0} \in L^{2}(\Omega) \cap W^{1, p}(\Omega) \text { and } \int_{\Omega} f\left(x, D u_{0}\right) \mathrm{d} x<\infty .
$$

We define variational solutions in the same way as in [1]:

Definition 1.1. Suppose that $f: \Omega \times \mathbb{R}^{n} \rightarrow \mathbb{R} \cup\{\infty\}$ is a variational integrand satisfying (1.1) and that the Cauchy-Dirichlet datum $u_{0}$ fulfills (1.2). We identify a measurable map $u: \Omega_{T} \rightarrow \mathbb{R}$ in the class

$$
u \in L^{p}\left(0, T ; W_{u_{0}}^{1, p}(\Omega)\right) \cap C^{0}\left([0, T], L^{2}(\Omega)\right)
$$

as a variational solution if and only if the variational inequality

$$
\begin{aligned}
\int_{0}^{T} \int_{\Omega} f(x, D u) \mathrm{d} x \mathrm{~d} t \leq & \int_{0}^{T} \int_{\Omega}\left[\partial_{t} v \cdot(v-u)+f(x, D v)\right] \mathrm{d} x \mathrm{~d} t \\
& +\frac{1}{2}\left\|v(\cdot, 0)-u_{0}\right\|_{L^{2}(\Omega)}^{2}-\frac{1}{2}\|(v-u)(\cdot, T)\|_{L^{2}(\Omega)}^{2}
\end{aligned}
$$

holds true, whenever $v \in L^{p}\left(0, T ; W_{u_{0}}^{1, p}(\Omega)\right)$ with $\partial_{t} v \in L^{2}\left(\Omega_{T}\right)$.

Here we used the shorthand notation

$$
W_{u_{0}}^{1, p}(\Omega):=u_{0}+W_{0}^{1, p}(\Omega)
$$

and later on we will use the abbreviations

$$
\begin{aligned}
& B_{\rho}\left(x_{0}\right):=\left\{x \in \mathbb{R}^{n}:\left|x-x_{0}\right|<\rho\right\}, \\
& Q_{\rho}\left(z_{0}\right):=B_{\rho}\left(x_{0}\right) \times\left(t_{0}-\rho^{2}, t_{0}\right),
\end{aligned}
$$

with $z_{0}=\left(x_{0}, t_{0}\right) \in \mathbb{R}^{n+1}$ and $x_{0} \in \mathbb{R}^{n}$. In this setting we will show: 
Theorem 1.2. Let

$$
u \in L^{p}\left(0, T ; W_{u_{0}}^{1, p}(\Omega)\right) \cap C^{0}\left([0, T] ; L^{2}(\Omega)\right)
$$

be a variational solution, where the variational integrand $f$ satisfies (1.1) and the initial datum $u_{0}$ fulfills (1.2). If

$$
\frac{2 n}{n+2}<p \leq q \leq p_{\star}=p \frac{n+2}{n}
$$

holds, then $u$ is locally bounded in $\Omega_{T}$. Moreover, if $q<p_{\star}$ holds, we have for any $\delta$ with $\max \{q, 2\} \leq \delta<p_{\star}$ and $Q_{\rho}\left(z_{0}\right) \Subset \Omega_{T}$

$$
\sup _{Q_{\frac{\rho}{2}}\left(z_{0}\right)}|u| \leq c\left(1+\rho^{-\delta \frac{n+p}{n}} \int_{Q_{\rho}\left(z_{0}\right)}|u|^{\delta} \mathrm{d} z\right)^{\frac{p}{n} \frac{1}{p_{\star}-\delta}}
$$

with a constant $c=c(n, p, q, L, \nu)$.

Remark 1.3. Note that the right-hand side is finite, since $u \in L^{p_{\star}}\left(\Omega_{T}\right)$ holds (c.f. Lemma 2.1).

It is also possible to show a comparable result in the sub-critical case $1<p \leq 2 n /(n+2)$, but we have to assume some higher integrability for $u$.

Theorem 1.4. Let

$$
1<p \leq \frac{2 n}{n+2} \quad \text { and } \quad r>\frac{n(2-p)}{p}
$$

If

$$
u \in L_{\mathrm{loc}}^{r}\left(\Omega_{T}\right) \cap L^{p}\left(0, T ; W_{u_{0}}^{1, p}(\Omega)\right) \cap C^{0}\left([0, T] ; L^{2}(\Omega)\right)
$$

is a variational solution, where the integrand $f$ satisfies (1.1), the initial datum $u_{0}$ fulfills (1.2) and

$$
p \leq q \leq p_{\star}=p \frac{n+2}{n}
$$

holds, then $u$ is locally bounded in $\Omega_{T}$. Additionally, if $q<p_{\star}$ holds, we have for any $Q_{\rho}\left(z_{0}\right) \Subset \Omega_{T}$

$$
\sup _{Q_{\frac{\rho}{2}}\left(z_{0}\right)} u \leq c\left(1+\rho^{-\frac{2(n+p)}{p}} \int_{Q_{\rho}\left(z_{0}\right)}|u|^{r} \mathrm{~d} z\right)^{\frac{p}{\lambda_{r}}},
$$

with $\lambda_{r}=n(p-2)+r p$ and $c=c(n, p, q, \nu, L, r)$.

Remark 1.5. The assumption $u \in L_{\text {loc }}^{r}\left(\Omega_{T}\right)$ is already needed and sharp in the case of parabolic equations with p-growth (cf. [6] Ch. 5). Otherwise, there are examples of unbounded weak solutions. The condition $r>n(2-p) / p$ guarantees that $\lambda_{r}>0$ holds. 


\subsection{Anisotropic variational integrals}

Here we consider local parabolic minimizers of evolutionary problems, where the integrand $f$ satisfies an anisotropic growth condition of the form

$$
\nu \sum_{i=1}^{n}\left|\zeta_{i}\right|^{p_{i}} \leq f(x, t, u, \zeta) \leq L\left(1+\sum_{i=1}^{n}\left|\zeta_{i}\right|^{p_{i}}\right)
$$

with $p_{i}>1$ and $p>1$ where

$$
\frac{1}{p}=\frac{1}{n} \sum_{i=1}^{n} \frac{1}{p_{i}}
$$

holds. Note that this is a special case of (1.1).

In this context, we define the anisotropic Sobolev space $W^{1, p_{i}}(\Omega)$ as the closure of $C^{\infty}(\Omega)$ under the norm

$$
\|u\|_{W^{1, p_{i}(\Omega)}}:=\sum_{i=1}^{n}\left\|D_{i} u\right\|_{L^{p_{i}(\Omega)}}+\|u\|_{L^{1}(\Omega)}
$$

and we use the same definition of local parabolic minimizers as in [17]:

Definition 1.6. A measurable map $u: \Omega_{T} \rightarrow \mathbb{R}$ is termed local parabolic minimizer associated to the variational integrand $f$ if and only if

$$
u \in L^{p}\left(0, T ; W^{1, p_{i}}(\Omega)\right) \cap C^{0}\left([0, T] ; L^{2}(\Omega)\right)
$$

and moreover, the following minimality condition

$$
\int_{\operatorname{spt} \varphi} u \cdot \partial_{t} \varphi+f(x, t, u, D u) \mathrm{d} z \leq \int_{\operatorname{spt} \varphi} f(x, t, u+\varphi, D u+D \varphi) \mathrm{d} z
$$

holds true, whenever $\varphi \in C_{0}^{\infty}\left(\Omega_{T}\right)$.

The appearance of this definition is natural in the context of variational solutions. In [1, Proposition 3.2] it is shown that every variational solution $u$ in the sense of Definition 1.1 also satisfies (1.6). Though the reverse statement is only true, if $\partial_{t} \in L^{2}\left(\Omega_{T}\right)$ or $\partial_{t} \in L^{p^{\prime}}\left(0, T ; W^{-1, p^{\prime}}(\Omega)\right)$ holds.

Now we formulate our results for anisotropic integrands.

Theorem 1.7. Let

$$
u \in L^{p}\left(0, T ; W^{1, p_{i}}(\Omega)\right) \cap C^{0}\left([0, T] ; L^{2}(\Omega)\right)
$$

be a parabolic minimizer, where the variational integrand satisfies (1.5). If

$$
\frac{2 n}{n+2}<p \quad \text { and } \quad p_{i} \leq p_{\star}=p \frac{n+2}{n} \text { for any } i \in\{1, \ldots, n\}
$$

holds, then $u$ is locally bounded in $\Omega_{T}$. Moreover, if $p_{i}<p_{\star}$ holds, we have for any $\delta$ with $\max \left\{p_{i}, 2\right\} \leq \delta<p_{\star}$ and $Q_{\rho}\left(z_{0}\right) \Subset \Omega_{T}$

$$
\sup _{Q_{\frac{\rho}{2}}\left(z_{0}\right)}|u| \leq c\left(1+\rho^{-\delta \frac{n+p}{n}} \int_{Q_{\rho}\left(z_{0}\right)}|u|^{\delta} \mathrm{d} z\right)^{\frac{p}{n} \frac{1}{p_{\star}-\delta}}
$$

with a constant $c=c\left(n, p_{i}, L, \nu\right)$. 
Again we have an analogous result in the sub-critical case.

Theorem 1.8. For any $i \in\{1, \ldots, n\}$ let

$$
1<p \leq \frac{2 n}{n+2}, \quad p_{i} \leq p_{\star}=p \frac{n+2}{n} \quad \text { and } \quad r>\frac{n(2-p)}{p} .
$$

If

$$
u \in L_{\mathrm{loc}}^{r}\left(\Omega_{T}\right) \cap L^{p}\left(0, T ; W^{1, p_{i}}(\Omega)\right) \cap C^{0}\left([0, T] ; L^{2}(\Omega)\right)
$$

is a parabolic minimizer, where the variational integrand satisfies (1.5), then $u$ is locally bounded in $\Omega_{T}$. Additionally, if $p_{i}<p_{\star}$ holds, we have for any $Q_{\rho}\left(z_{0}\right) \Subset \Omega_{T}$

$$
\sup _{Q_{\frac{\rho}{2}}\left(z_{0}\right)} u \leq c\left(1+\rho^{-\frac{2(n+p)}{p}} \int_{Q_{\rho}\left(z_{0}\right)}|u|^{r} \mathrm{~d} z\right)^{\frac{p}{\lambda_{r}}},
$$

with $\lambda_{r}=n(p-2)+r p$ and $c=c\left(n, p_{i}, q, \nu, L, r\right)$.

\section{Preliminaries}

\subsection{Auxiliary tools}

In this subsection we state several auxiliary tools, that will be needed throughout the paper. We start with a parabolic version of the Sobolev embedding (cf. [6, Ch. 1, Proposition 3.1]).

Lemma 2.1. Let $u \in C^{0}\left([0, T] ; L^{2}(\Omega)\right) \cap L^{p}\left(0, T ; W_{0}^{1, p}(\Omega)\right)$. Then there exists a constant $c$ depending only on $n$ and $p$ such that

$$
\left(\int_{\Omega_{T}}|u|^{p_{\star}} \mathrm{d} z\right)^{\frac{n}{n+p}} \leq c\left(\sup _{0<t<T} \int_{\Omega}|u(\cdot, t)|^{2} \mathrm{~d} x+\int_{\Omega_{T}}|D u|^{p} \mathrm{~d} z\right),
$$

with $p_{\star}=p \frac{n+2}{n}$.

We also need an anisotropic version of the last Lemma ([15, Lemma 1]):

Lemma 2.2. Let $u \in C^{0}\left([0, T] ; L^{2}(\Omega)\right) \cap L^{p}\left(0, T ; W_{0}^{1, p_{i}}(\Omega)\right)$ and suppose that (1.7) holds. Then there exists a constant $c$ depending only on $n$ and $p_{i}$ such that

$$
\left(\int_{\Omega_{T}}|u|^{p_{\star}} \mathrm{d} z\right)^{\frac{n}{n+p}} \leq c\left(\sup _{0<t<T} \int_{\Omega}|u(\cdot, t)|^{2} \mathrm{~d} x+\int_{\Omega_{T}} \sum_{i=1}^{n}\left|D_{i} u\right|^{p_{i}} \mathrm{~d} z\right),
$$

with $p_{\star}=p \frac{n+2}{n}$.

Furthermore, we will use the following well known Lemmata (cf. [11, Lemma 4.7, Ch.II], [6, Lemma 4.3, Ch. I] and [9, Lemma 1.1]):

Lemma 2.3. Let $\left\{J_{h}\right\}_{h \in \mathbb{N}_{0}}$ be a sequence of non-negative numbers, verifying the condition

$$
J_{h+1} \leq M b^{h} J_{h}^{1+\gamma}
$$


for $h \in \mathbb{N}_{0}$, where $M, \gamma$ and $b$ are positive constants and $b>1$. Then:

$$
J_{h} \leq M^{\frac{(1+\gamma)^{h}-1}{\gamma}} b^{\frac{(1+\gamma)^{h}-1}{\gamma^{2}}-\frac{h}{\gamma}} J_{0}^{(1+\gamma)^{h}} .
$$

In particular, if

$$
J_{0} \leq \theta=M^{-\frac{1}{\gamma}} b^{-\frac{1}{\gamma^{2}}}
$$

then

$$
J_{h} \leq \theta b^{-\frac{h}{\gamma}}
$$

and therefore $J_{h} \rightarrow 0$ as $h \rightarrow \infty$.

Lemma 2.4. Let $\left\{M_{h}\right\}_{h \in \mathbb{N}_{0}}$ be a sequence of equibounded positive numbers satisfying the recursive inequalities

$$
M_{h} \leq C b^{h} M_{h+1}^{1-\alpha},
$$

where $C, b>1$ and $\alpha \in(0,1)$ are given constants. Then

$$
M_{0} \leq\left(\frac{2 C}{b^{1-\frac{1}{\alpha}}}\right)^{\frac{1}{\alpha}}
$$

holds.

Lemma 2.5. Let $f(t)$ be a non-negative bounded function defined for $0 \leq T_{1} \leq$ $t \leq T_{2}$. Suppose that for $T_{1} \leq s<t \leq T_{2}$ we have

$$
f(s) \leq \theta f(t)+A(t-s)^{-\alpha}+B,
$$

where $A, B, \alpha, \theta$ are positive constants with $\theta<1$. Then there exists a constant $c$, depending only on $\alpha$ and $\theta$ such that for every $T_{1} \leq \rho<R \leq T_{2}$ we have

$$
f(\rho) \leq c\left[A(R-\rho)^{-\alpha}+B\right] .
$$

\subsection{Time derivative}

Now we prove the existence of the time derivative in $L^{2}\left(\Omega_{T}\right)$ of variational solutions, if they possess time independent boundary values (cf. [2]). Therefore, we only need the convexity of the integrand $f$. To be more precise, we have:

Lemma 2.6. Let $u \in C^{0}\left([0, T], L^{2}(\Omega)\right) \cap L^{p}\left(0, T ; W_{u_{0}}^{1, p}(\Omega)\right)$ be a variational solution in the sense of Definition (1.1), where the initial datum $u_{0}$ satisfies (1.2) and $f(x, \zeta)$ is convex with respect to $\zeta$. Then we have $\partial_{t} u \in L^{2}\left(\Omega_{T}\right)$.

Proof. We will use the mollification in time

$$
[u]_{h}(\cdot, t)=e^{-\frac{t}{h}} u_{0}+\frac{1}{h} \int_{0}^{t} e^{\frac{s-t}{h}} u(\cdot, s) \mathrm{d} s .
$$

For more properties of this mollification see [10, Lemma 2.2] and [1, Lemma $2.2,2.3]$. Now we can test the variational inequality with $v=[u]_{h}$. If we use $[u]_{h}(\cdot, 0)=u_{0}$, we obtain

$$
\int_{0}^{T} \int_{\Omega} f(x, D u) \mathrm{d} x \mathrm{~d} t \leq \int_{0}^{T} \partial_{t}[u]_{h} \cdot\left([u]_{h}-u\right)+f\left(x, D[u]_{h}\right) \mathrm{d} x \mathrm{~d} t .
$$


But this implies

$$
\begin{aligned}
\int_{0}^{T} \int_{\Omega}\left|\partial_{t}[u]_{h}\right|^{2} \mathrm{~d} x \mathrm{~d} t & =-\int_{0}^{T} \int_{\Omega} \frac{1}{h} \partial_{t}[u]_{h} \cdot\left([u]_{h}-u\right) \mathrm{d} x \mathrm{~d} t \\
& \leq \frac{1}{h} \int_{0}^{T} \int_{\Omega} f\left(x, D[u]_{h}\right)-f(x, D u) \mathrm{d} x \mathrm{~d} t \\
& \leq \frac{1}{h} \int_{0}^{T} \int_{\Omega}[f(x, D u)]_{h}-f(x, D u) \mathrm{d} x \mathrm{~d} t \\
& =-\int_{0}^{T} \int_{\Omega} \partial_{t}[f(x, D u)]_{h} \mathrm{~d} x \mathrm{~d} t \\
& =\int_{\Omega}\left[f\left(x, D u_{0}\right)\right]_{h}-[f(x, D u(\cdot, T))]_{h} \mathrm{~d} x \\
& \leq \int_{\Omega} f\left(x, D u_{0}\right) \mathrm{d} x<\infty
\end{aligned}
$$

Hence $\partial_{t} u \in L^{2}\left(\Omega_{T}, \mathbb{R}^{N}\right)$ holds. Note that we used

$$
\partial_{t}[u]_{h}=-\frac{1}{h}\left([u]_{h}-u\right)
$$

and

$$
f\left(x, D[u]_{h}\right) \leq[f(x, D u)]_{h},
$$

which holds due to the convexity of $f$, cf. [1, Lemma 2.3].

\section{3. $L_{\text {loc }}^{\infty}$-bound for $p, q$-integrands}

In this section we show the $L_{\mathrm{loc}}^{\infty}$-bound for variational solutions stated in Theorems 1.2 and 1.4. First, we will only consider the case $q<p(n+2) / n$ and give an explicit $L^{\infty}$-bound for $u$. In Sect. 5 we will treat the case $q=p(n+2) / n$. In order to prove our results, we want to argue on the level of parabolic minimizers. Therefore we use the following definition:

Definition 3.1. A measurable map $u: \Omega_{T} \rightarrow \mathbb{R}$ is termed parabolic minimizer associated to the variational integrand $f$ and the Cauchy-Dirichlet datum $u_{0}$ if and only if

$$
u \in L^{p}\left(0, T ; W_{u_{0}}^{1, p}(\Omega)\right)
$$

and moreover, the following minimality condition

$$
\int_{0}^{T} \int_{\Omega} u \cdot \partial_{t} \varphi+f(x, D u) \mathrm{d} x \mathrm{~d} t \leq \int_{0}^{T} \int_{\Omega} f(x, D u+D \varphi) \mathrm{d} x \mathrm{~d} t
$$

holds true, whenever $\varphi \in C_{0}^{\infty}\left(\Omega_{T}\right)$.

In [1, Proposition 3.2], it was shown, that every variational solution in the sense of Definition 1.1 is also a parabolic minimizer in the sense of Definition 3.1. The reverse statement is only true if $\partial_{t} u \in L^{2}\left(\Omega_{T}\right)$ or $\partial_{t} u \in$ $L^{p^{\prime}}\left(0, T ; W^{-1, p^{\prime}}(\Omega)\right)$ holds. Hence, it is not restrictive to use the definition of 
parabolic minimizer to show regularity for variational solutions. Our first aim is to prove a Caccioppoli inequality on superlevel sets

$$
A(k, \rho, \theta):=A\left(k, \rho, \theta ; z_{0}\right):=\left\{(x, t) \in Q_{\rho, \theta}\left(z_{0}\right): u(x, t)>k\right\},
$$

with

$$
Q_{\rho, \theta}\left(z_{0}\right):=B_{\rho}\left(x_{0}\right) \times\left(t_{0}-\theta, t_{0}\right)
$$

and $z_{0}=\left(x_{0}, t_{0}\right) \in \mathbb{R}^{n+1}$ and $k, \rho, \theta>0$. A crucial point in the proof is, that we can not use the "hole-filling technique" due to the growth conditions. To overcome this problem, we will use the convexity of $f$. A similar technique has been used in [7, Lemma 3.1] to prove a Caccioppoli inequality.

Lemma 3.2. Let $u \in C^{0}\left([0, T] ; L^{2}(\Omega)\right) \cap L^{p}\left(0, T ; W_{u_{0}}^{1, p}(\Omega)\right)$ be a variational solution, where the integrand $f$ satisfies (1.1) and the initial datum $u_{0}$ fulfills (1.2). Then for all cylinders $Q_{\rho, \rho^{2}}\left(z_{0}\right) \Subset \Omega_{T}$ and $k, \tau_{1}, \tau_{2}>0$ with $\frac{1}{2} \rho \leq \rho_{1}<$ $\rho_{2} \leq \rho, t_{0}-\rho^{2} \leq t_{0}-\tau_{2}<t_{0}-\tau_{1} \leq t_{0}-\frac{1}{2} \rho^{2}$ and $q \leq \delta \leq p_{\star}$ we have

$$
\begin{aligned}
& \sup _{t_{0}-\tau_{1}<t<t_{0}} \int_{B_{\rho_{1}}\left(x_{0}\right)}\left|(u-k)_{+}(\cdot, t)\right|^{2} \mathrm{~d} x+\int_{A\left(k, \rho_{1}, \tau_{1}\right)}|D u|^{p} \mathrm{~d} z \\
& \leq c \int_{A\left(k, \rho_{2}, \tau_{2}\right)} \frac{\left|(u-k)_{+}\right|^{2}}{\tau_{2}-\tau_{1}}+\frac{\left|(u-k)_{+}\right|^{\delta}}{\left(\rho_{2}-\rho_{1}\right)^{\delta}}+1 \mathrm{~d} z
\end{aligned}
$$

with a constant $c=c(p, q, \nu, L)$.

Proof. Since every variational solution is also a parabolic minimizer, we have for any $\varphi \in C_{0}^{\infty}\left(\Omega_{T}\right)$

$$
\int_{\operatorname{spt} \varphi} u \cdot \partial_{t} \varphi+f(x, D u) \mathrm{d} z \leq \int_{\operatorname{spt} \varphi} f(x, D u+D \varphi) \mathrm{d} z .
$$

For $k>0$ we choose

$$
\varphi=-\chi_{\varepsilon} \psi^{q} \zeta^{q}(u-k)_{+}
$$

as testing function, where the functions $\psi \in C^{1}\left(\left(t_{0}-\rho^{2}, t_{0}\right)\right)$ and $\zeta \in C_{0}^{1}\left(B_{\rho}\right)$ are cut-off functions with $0 \leq \psi, \zeta \leq 1$. Additionally we can choose $\zeta$ such that $\zeta \equiv 1$ on $B_{\rho_{1}}\left(x_{0}\right), \zeta \equiv 0$ outside of $\overline{B_{\rho_{2}}\left(x_{0}\right)}$ and $0 \leq|D \zeta| \leq 2 /\left(\rho_{2}-\rho_{1}\right)$ holds. For $\psi$ we can assume that $\psi \equiv 0$ on $\left(t_{0}-\rho^{2}, t_{0}-\tau_{2}\right), \psi \equiv 1$ on $\left(t_{0}-\tau_{1}, t_{0}\right)$ and $0 \leq \psi^{\prime} \leq 2 /\left(\tau_{2}-\tau_{1}\right)$ holds. Last, the function $\chi_{\varepsilon}$ is defined by

$$
\chi_{\varepsilon}:= \begin{cases}1 & t_{0}-\rho^{2} \leq t \leq \tau \\ 1-\frac{1}{\varepsilon}(t-\tau) & \tau<t<\tau+\varepsilon \\ 0 & \text { otherwise }\end{cases}
$$

for $\tau \in\left(t_{0}-\tau_{1}, t_{0}-\varepsilon\right)$ and $0<\varepsilon \ll 1$. From Lemma 2.6 we know that $\partial_{t} u \in L^{2}\left(\Omega_{T}\right)$ holds, because the solutions have time-independent boundary values. Hence we can take $\varphi$ as testing function, since this function can be approximated by smooth functions with compact support in $\Omega_{T}$. Now we use 
the convexity of $f$ to estimate the right hand side of (3.1)

$$
\begin{aligned}
\int_{\operatorname{spt} \varphi} f(x, D u+D \varphi) \mathrm{d} z & =\int_{\operatorname{spt} \varphi} f\left(x,\left(1-\psi^{q}\right) D u+\psi^{q}(D u+D \tilde{\varphi})\right) \mathrm{d} z \\
& \leq \int_{\operatorname{spt} \varphi}\left(1-\psi^{q}\right) f(x, D u)+\psi^{q} f(x, D u+D \tilde{\varphi}) \mathrm{d} z
\end{aligned}
$$

where we used the abbreviation

$$
\tilde{\varphi}:=-\chi_{\varepsilon} \zeta^{q}(u-k)_{+} .
$$

If we use this estimate in $(3.1)$ and subtract $\int_{\operatorname{spt} \varphi}\left(1-\psi^{q}\right) f(x, D u) \mathrm{d} z$ on both sides, we achieve

$$
\int_{\operatorname{spt} \varphi} u \cdot \partial_{t} \varphi+\psi^{q} f(x, D u) \mathrm{d} z \leq \int_{\operatorname{spt} \varphi} \psi^{q} f(x, D u+D \tilde{\varphi}) \mathrm{d} z
$$

Note that $\int_{\operatorname{spt} \varphi} f(x, D u) \mathrm{d} z$ is finite, since $u$ is a parabolic minimizer. Thus we were able to absorb this term. Next we estimate the right hand side, again using the convexity of $f$

$$
\begin{aligned}
\int_{\operatorname{spt} \varphi} & \psi^{q} f(x, D u+D \tilde{\varphi}) \mathrm{d} z \\
= & \int_{\operatorname{spt} \varphi} \psi^{q} f\left(x, D u-D\left(\chi_{\varepsilon} \zeta^{q}(u-k)_{+}\right)\right) \mathrm{d} z \\
= & \int_{\operatorname{spt} \varphi} \psi^{q} f\left(x, D(u-k)_{+}-q(u-k)_{+} \chi_{\varepsilon} \zeta^{q-1} D \zeta-\chi_{\varepsilon} \zeta^{q} D(u-k)_{+}\right) \mathrm{d} z \\
= & \int_{\operatorname{spt} \varphi} \psi^{q} f\left(x,\left(1-\chi_{\varepsilon} \zeta^{q}\right) D(u-k)_{+}+\chi_{\varepsilon} \zeta^{q}\left(-q \frac{(u-k)_{+}}{\zeta} D \zeta\right)\right) \mathrm{d} z \\
\leq & \int_{\operatorname{spt} \varphi} \psi^{q}\left(1-\chi_{\varepsilon} \zeta^{q}\right) f(x, D u)+\chi_{\varepsilon} \psi^{q} \zeta^{q} f\left(x,-q \frac{(u-k)_{+}}{\zeta} D \zeta\right) \mathrm{d} z .
\end{aligned}
$$

Putting this into (3.2) and subtracting $\int_{\operatorname{spt} \varphi} \psi^{q}\left(1-\chi_{\varepsilon} \zeta^{q}\right) f(x, D u) \mathrm{d} z$ on both sides, we obtain

$$
\begin{aligned}
& \int_{\operatorname{spt} \varphi} u \cdot \partial_{t} \varphi+\chi_{\varepsilon} \psi^{q} \zeta^{q} f(x, D u) \mathrm{d} z \\
& \quad \leq \int_{\operatorname{spt} \varphi} \chi_{\varepsilon} \psi^{q} \zeta^{q} f\left(x,-q \frac{(u-k)_{+}}{\zeta} D \zeta\right) \mathrm{d} z \\
& \quad \leq L \int_{\operatorname{spt} \varphi} \chi_{\varepsilon} \psi^{q} \zeta^{q} q^{q} \frac{\left|(u-k)_{+}\right|^{q}}{\zeta^{q}}|D \zeta|^{q}+\chi_{\varepsilon} \psi^{q} \zeta^{q} \mathrm{~d} z \\
& \leq c(L, q) \int_{\operatorname{spt} \varphi}\left|(u-k)_{+}\right|^{q}|D \zeta|^{q}+1 \mathrm{~d} z .
\end{aligned}
$$


Note that we used the growth-condition (1.1). Now we consider the term, that involves the time derivative and compute

$$
\begin{aligned}
\int_{\operatorname{spt} \varphi} u \cdot \partial_{t} \varphi \mathrm{d} z & \\
= & -\int_{A\left(k, \rho_{2}, \tau_{2}\right)} \partial_{t} u \cdot \varphi \mathrm{d} z \\
= & \int_{A\left(k, \rho_{2}, \tau_{2}\right)} \partial_{t}(u-k)_{+} \chi_{\varepsilon} \psi^{q} \zeta^{q}(u-k)_{+} \mathrm{d} z \\
= & \int_{A\left(k, \rho_{2}, \tau_{2}\right)} \frac{1}{2} \partial_{t}\left|(u-k)_{+}\right|^{2} \chi_{\varepsilon} \psi^{q} \zeta^{q} \mathrm{~d} z \\
= & -\int_{A\left(k, \rho_{2}, \tau_{2}\right)} \frac{1}{2}\left|(u-k)_{+}\right|^{2}\left(\chi_{\varepsilon}^{\prime} \psi^{q} \zeta^{q}+q \chi_{\varepsilon} \psi^{q-1} \psi^{\prime} \zeta^{q}\right) \mathrm{d} z \\
& \stackrel{\varepsilon \downarrow 0}{\longrightarrow} \int_{B_{\rho_{2}}\left(x_{0}\right)} \zeta^{q}\left|(u-k)_{+}(\cdot, \tau)\right|^{2} \mathrm{~d} x \\
& -\frac{q}{2} \int_{A\left(k, \rho_{2}, \tau_{2}\right)}\left|(u-k)_{+}\right|^{2} \psi^{q-1} \psi^{\prime} \zeta^{q} \chi_{\left(t_{0}-\tau_{1}, \tau\right)} \\
\geq & \frac{1}{2} \int_{B_{\rho_{1}}\left(x_{0}\right)}\left|(u-k)_{+}(\cdot, \tau)\right|^{2} \mathrm{~d} x-q \int_{A\left(k, \rho_{2}, \tau_{2}\right)} \frac{\left|(u-k)_{+}\right|^{2}}{\tau_{2}-\tau_{1}} \mathrm{~d} z .
\end{aligned}
$$

If we let $\varepsilon \downarrow 0$ in (3.3) and insert the last estimate, take the supremum over $\tau \in\left(t_{0}-\tau_{1}, t_{0}\right)$ in the first term on the left hand side, let $\tau \rightarrow t_{o}-\tau_{1}$ in the second term on the left hand side and use the growth assumption (1.1), we get

$$
\begin{aligned}
& \sup _{t \in\left(t_{0}-\tau_{1}, t_{0}\right)} \int_{B_{\rho_{1}}\left(x_{0}\right)}\left|(u-k)_{+}(\cdot, t)\right|^{2} \mathrm{~d} x+\int_{A\left(k, \rho_{1}, \tau_{1}\right)}|D u|^{p} \mathrm{~d} z \\
& \leq c \int_{A\left(k, \rho_{2}, \tau_{2}\right)} \frac{\left|(u-k)_{+}\right|^{2}}{\tau_{2}-\tau_{1}}+\frac{\left|(u-k)_{+}\right|^{q}}{\left(\rho_{2}-\rho_{1}\right)^{q}}+1 \mathrm{~d} z \\
& \leq c \int_{A\left(k, \rho_{2}, \tau_{2}\right)} \frac{\left|(u-k)_{+}\right|^{2}}{\tau_{2}-\tau_{1}}+\frac{\left|(u-k)_{+}\right|^{\delta}}{\left(\rho_{2}-\rho_{1}\right)^{\delta}}+1 \mathrm{~d} z,
\end{aligned}
$$

with a constant $c$ depending only on $p, q, \nu$ and $L$.

Now we are ready to start with the proof of Theorem 1.2. Here we will only show the $L_{\mathrm{loc}}^{\infty}$-bound for the case $q<p_{\star}$. The limit case $q=p_{\star}$ will be treated in Sect. 5 .

Proof of Theorem 1.2. Consider a parabolic cylinder $Q_{\rho, \rho^{2}}\left(z_{0}\right) \Subset \Omega_{T}$ and $k \geq$ 1. For $h \in \mathbb{N}_{0}$ we define

$$
\left\{\begin{array}{l}
\rho_{h}:=\frac{\rho}{2}+\frac{\rho}{2^{h+1}}, \bar{\rho}_{h}:=\frac{\rho_{h}+\rho_{h+1}}{2} \\
\tau_{h}:=\left(\frac{\rho^{2}}{4}+3 \frac{\rho^{2}}{4^{h+1}}\right), \bar{\tau}_{h}:=\frac{\tau_{h}+\tau_{h+1}}{2} \\
k_{h}:=k\left(1-\frac{1}{2^{h}}\right)
\end{array}\right.
$$


and

$$
J_{h}:=\int_{A\left(k_{h}, \rho_{h}, \tau_{h}\right)}\left|u-k_{h}\right|^{\delta} \mathrm{d} z .
$$

Now we choose $\psi_{h} \in C^{1}\left(\left(t_{0}-\rho^{2}, t_{0}\right)\right)$ and $\zeta_{h} \in C_{0}^{1}\left(B_{\rho}\left(x_{0}\right)\right)$ with $0 \leq \zeta_{h} \leq 1$ and $\zeta_{h} \equiv 1$ in $B_{\rho_{h+1}}\left(x_{0}\right), \zeta_{h} \equiv 0$ outside of $B_{\bar{\rho}_{h}}\left(x_{0}\right)$ and $\left|D \zeta_{h}\right| \leq \frac{2^{h}}{\rho}$. Further we take $0 \leq \psi_{h} \leq 1$ with $\psi_{h} \equiv 1$ in $\left(t_{0}-\tau_{h+1}, t_{0}\right)$ and $\psi_{h} \equiv 0$ for $t \leq t_{0}-\bar{\tau}_{h}$. With these choices and the Lemmas 2.1 and 3.2, we have for $\max \{q, 2\} \leq \delta<p_{\star}$

$$
\begin{aligned}
& J_{h+1} \leq \int_{A\left(k_{h+1}, \bar{\rho}_{h}, \bar{\tau}_{h}\right)}\left|\left(u-k_{h+1}\right) \psi_{h} \zeta_{h}\right|^{\delta} \mathrm{d} z \\
& \leq\left(\int_{A\left(k_{h+1}, \bar{\rho}_{h}, \bar{\tau}_{h}\right)}\left|\left(u-k_{h+1}\right) \psi_{h} \zeta_{h}\right|^{p_{\star}} \mathrm{d} z\right)^{\frac{\delta}{p_{\star}}} \cdot\left|A\left(k_{h+1}, \bar{\rho}_{h}, \bar{\tau}_{h}\right)\right|^{1-\frac{\delta}{p_{\star}}} \\
& \leq c\left[\sup _{t_{0}-\bar{\tau}_{h}<t<t_{0}} \int_{B_{\bar{\rho}_{h}}\left(x_{0}\right)}\left|\left(u-k_{h+1}\right)_{+}(\cdot, t) \psi_{h}(t) \zeta_{h}\right|^{2} \mathrm{~d} x\right. \\
& \left.+\int_{A\left(k_{h+1}, \bar{\rho}_{h}, \bar{\tau}_{h}\right)}\left|D\left[\left(u-k_{h+1}\right) \psi_{h} \zeta_{h}\right]\right|^{p} \mathrm{~d} z\right] \cdot\left|A\left(k_{h+1}, \rho_{h}, \tau_{h}\right)\right|^{1-\frac{\delta}{p}} \text { * } \\
& \leq c\left[\sup _{t_{0}-\bar{\tau}_{h}<t<t_{0}} \int_{B_{\bar{\rho}_{h}}\left(x_{0}\right)}\left|\left(u-k_{h+1}\right)_{+}(\cdot, t)\right|^{2} \mathrm{~d} x+\int_{A\left(k_{h+1}, \bar{\rho}_{h}, \bar{\tau}_{h}\right)}|D u|^{p} \mathrm{~d} z\right. \\
& \left.+\int_{A\left(k_{h+1}, \bar{\rho}_{h}, \bar{\tau}_{h}\right)} \frac{2^{h p}}{\rho^{p}}\left|u-k_{h+1}\right|^{p} \mathrm{~d} z\right]^{\frac{n+p}{n} \frac{\delta}{p} \star} \cdot\left|A\left(k_{h+1}, \rho_{h}, \tau_{h}\right)\right|^{1-\frac{\delta}{p}} \star \\
& \leq c\left[\int_{A\left(k_{h+1}, \rho_{h}, \tau_{h}\right)} \frac{2^{h \delta}}{\rho^{\delta}}\left|u-k_{h+1}\right|^{\delta}+\frac{4^{h}}{\rho^{2}}\left|u-k_{h+1}\right|^{2}+1 \mathrm{~d} z\right]^{\frac{n+p}{n} \frac{\delta}{p}} \text { * } \\
& \times\left|A\left(k_{h+1}, \rho_{h}, \tau_{h}\right)\right|^{1-\frac{\delta}{p}} \text { * } \\
& \leq c\left[\frac{2^{h \delta}}{\rho^{\delta}} \int_{A\left(k_{h+1}, \rho_{h}, \tau_{h}\right)}\left|u-k_{h+1}\right|^{\delta}+1 \mathrm{~d} z\right]^{\frac{n+p}{n} \frac{\delta}{p_{\star}}} \cdot\left|A\left(k_{h+1}, \rho_{h}, \tau_{h}\right)\right|^{1-\frac{\delta}{p_{\star}}} .
\end{aligned}
$$

Now we observe that

$$
\int_{A\left(k_{h+1}, \rho_{h}, \tau_{h}\right)}\left|u-k_{h+1}\right|^{\delta} \mathrm{d} z \leq J_{h}
$$

and

$$
\left|A\left(k_{h+1}, \rho_{h}, \tau_{h}\right)\right|\left(k_{h+1}-k_{h}\right)^{\delta} \leq \int_{A\left(k_{h+1}, \rho_{h}, \tau_{h}\right)}\left(u-k_{h}\right)^{\delta} \mathrm{d} z \leq J_{h}
$$

and since $k \geq 1$, we have

$$
\left|A\left(k_{h+1}, \rho_{h}, \tau_{h}\right)\right| \leq \frac{4^{(h+2) \delta}}{k^{\delta}} J_{h} \leq 4^{(h+2) \delta} J_{h} .
$$


Then, (3.6) turns into

$$
\begin{aligned}
J_{h+1} & \leq c\left[\frac{2^{h \delta}}{\rho^{\delta}} J_{h}\right]^{\frac{n+p}{n} \frac{\delta}{p}}\left(\frac{4^{h \delta}}{k^{\delta}} J_{h}\right)^{1-\frac{\delta}{p}}{ }^{\frac{c}{k^{\frac{\delta}{p_{\star}}}\left(p_{\star}-\delta\right)} \rho^{\delta \frac{n+p}{n} \frac{\delta}{p_{\star}}}}\left(4^{\delta\left(1+\frac{\delta}{p_{\star}} \frac{p}{n}\right)}\right)^{h} J_{h}^{1+\frac{p}{n} \frac{\delta}{p_{\star}}}
\end{aligned}
$$

with $c=c(n, p, q, L, \nu)$. Now we use Lemma 2.3 with

$$
\gamma=\frac{p}{n} \frac{\delta}{p_{\star}}, b=4^{\delta\left(1+\frac{\delta}{p_{\star}} \frac{p}{n}\right)} \text { and } M=\frac{c}{k^{\frac{\delta}{p_{\star}}\left(p_{\star}-\delta\right)} \rho^{\delta \frac{n+p}{n} \frac{\delta}{p_{\star}}}} .
$$

If we choose $k \geq 1$ such that

$$
\int_{Q_{\rho, \rho^{2}}\left(z_{0}\right) \cap\{u>0\}}|u|^{\delta} \mathrm{d} z=J_{0} \leq M^{-\frac{1}{\gamma}} b^{-\frac{1}{\gamma^{2}}},
$$

we get $\lim _{h \rightarrow \infty} J_{h}=0$ and thus

$$
\sup _{\frac{\rho}{2}, \frac{\rho^{2}}{4}\left(z_{0}\right)} u \leq k
$$

or

$$
\sup _{Q_{\frac{\rho}{2}}\left(z_{0}\right)} u \leq c\left(1+\rho^{-\delta \frac{n+p}{n}} \int_{Q_{\rho}\left(z_{0}\right)}|u|^{\delta} \mathrm{d} z\right)^{\frac{p}{n} \frac{1}{p_{\star}-\delta}}
$$

with $c=c(n, p, q, L, \nu)$. Now we have proved that $u$ is locally bounded from above in $\Omega_{T}$. Moreover, $-u$ is a local minimizer of the integrand $\tilde{f}(x, \zeta):=$ $f(x,-\zeta)$. Since $\tilde{f}$ satisfies the same growth conditions as $f$, we conclude that $-u$ is locally bounded from above by the same bound and the proof is completed for $q<p_{\star}$.

Now we will prove Theorem 1.4. Therefore, we let $1<p \leq 2 n /(n+2)$. Since we are in the sup-critical case we need to assume that $u$ is integrable with exponent $r>n(2-p) / p$ in order to show the $L_{\mathrm{loc}}^{\infty}$-bound. We also note, that we can use Lemma 3.2, since we do not require any assumptions on $p$ there. Now we will only consider the case $p<p_{\star}$, for the limit case we refer to Sect. 5 .

Proof of Theorem 1.4. Let $Q_{\rho, \rho^{2}}\left(z_{0}\right) \Subset \Omega_{T}$ and $k \geq 1$. First we notice that $p_{\star}=p(n+2) / n \leq 2$ holds. We define $\rho_{h}, \bar{\rho}_{h}, \tau_{h}, \bar{\tau}_{h}$ and $k_{h}$ as in (3.4) and set

$$
J_{h}:=\int_{A\left(k_{h}, \rho_{h}, \tau_{h}\right)}\left|\left(u-k_{h}\right)\right|^{2} \mathrm{~d} z .
$$

Now we choose $\psi_{h} \in C^{1}\left(\left(t_{0}-\rho^{2}, t_{0}\right)\right)$ and $\zeta_{h} \in C_{0}^{1}\left(B_{\rho}\left(x_{0}\right)\right)$ with $0 \leq \zeta_{h} \leq 1$ and $\zeta_{h} \equiv 1$ in $B_{\rho_{h+1}}\left(x_{0}\right), \zeta_{h} \equiv 0$ outside of $B_{\bar{\rho}_{h}}\left(x_{0}\right)$ and $\left|D \zeta_{h}\right| \leq \frac{2^{h}}{\rho}$. Further 
we take $0 \leq \psi_{h} \leq 1$ with $\psi_{h} \equiv 1$ in $\left(t_{0}-\tau_{h+1}, t_{0}\right)$ and $\psi_{h} \equiv 0$ for $t \leq t_{0}-\bar{\tau}_{h}$ we obtain

$$
\begin{aligned}
J_{h+1} \leq & \int_{A\left(k_{h+1}, \bar{\rho}_{h}, \bar{\tau}_{h}\right)}\left|\left(u-k_{h+1}\right) \psi_{h} \zeta_{h}\right|^{2} \mathrm{~d} z \\
\leq & \left(\int_{A\left(k_{h+1}, \bar{\rho}_{h}, \bar{\tau}_{h}\right)}\left|\left(u-k_{h+1}\right) \psi_{h} \zeta_{h}\right|^{q} \mathrm{~d} z\right)^{\frac{2 \alpha}{q}} \\
& \times\left(\int_{A\left(k_{h+1}, \bar{\rho}_{h}, \bar{\tau}_{h}\right)}\left|\left(u-k_{h+1}\right) \psi_{h} \zeta_{h}\right|^{r} \mathrm{~d} z\right)^{\frac{2(1-\alpha)}{r}},
\end{aligned}
$$

for $\alpha \in(0,1)$ with

$$
1=\frac{2 \alpha}{q}+\frac{2(1-\alpha)}{r}
$$

With the abbreviation

$$
M:=\left(\int_{Q_{\rho}\left(z_{0}\right)}|u|^{r} \mathrm{~d} z\right)^{\frac{2(1-\alpha)}{r}}
$$

and Lemmas 2.1 and 3.2 (with $\delta=q$ ), inequality (3.10) turns into

$$
\begin{aligned}
& J_{h+1} \leq\left(\int_{A\left(k_{h+1}, \bar{\rho}_{h}, \bar{\tau}_{h}\right)}\left|\left(u-k_{h+1}\right) \psi_{h} \zeta_{h}\right|^{q} \mathrm{~d} z\right)^{\frac{2 \alpha}{q}} \\
& \times\left(\int_{A\left(k_{h+1}, \bar{\rho}_{h}, \bar{\tau}_{h}\right)}\left|\left(u-k_{h+1}\right) \psi_{h} \zeta_{h}\right|^{r} \mathrm{~d} z\right)^{\frac{2(1-\alpha)}{r}} \\
& \leq M\left(\int_{A\left(k_{h+1}, \bar{\rho}_{h}, \bar{\tau}_{h}\right)}\left|\left(u-k_{h+1}\right) \psi_{h} \zeta_{h}\right|^{q} \mathrm{~d} z\right)^{\frac{2 \alpha}{q}} \\
& \leq M\left(\left(\int_{A\left(k_{h+1}, \bar{\rho}_{h}, \bar{\tau}_{h}\right)}\left|\left(u-k_{h+1}\right) \psi_{h} \zeta_{h}\right|^{p_{\star}}\right)^{\frac{q}{p_{\star}}}\left|A\left(k_{h+1}, \bar{\rho}_{h}, \bar{\tau}_{h}\right)\right|^{1-\frac{q}{p_{\star}}}\right)^{\frac{2 \alpha}{q}} \\
& \leq c M\left(\left[\sup _{t_{0}-\bar{\tau}_{h}<t<t_{0}} \int_{K\left(\bar{\rho}_{h}\right)}\left|\left(u-k_{h+1}\right)_{+}(\cdot, t)\right|^{2} \mathrm{~d} x+\int_{A\left(k_{h+1}, \bar{\rho}_{h}, \bar{\tau}_{h}\right)}|D u|^{p} \mathrm{~d} z\right.\right. \\
& \left.\left.+\int_{A\left(k_{h+1}, \bar{\rho}_{h}, \bar{\tau}_{h}\right)} \frac{4^{h p}}{\rho^{p}}\left|u-k_{h+1}\right|^{p} \mathrm{~d} z\right]^{\frac{n+p}{n} \frac{q}{p_{\star}}} \cdot\left|A\left(k_{h+1}, \rho_{h}, \tau_{h}\right)\right|^{1-\frac{q}{p_{\star}}}\right)^{\frac{2 \alpha}{q}}
\end{aligned}
$$




$$
\begin{aligned}
& \leq c M\left(\left[\int_{A\left(k_{h+1}, \rho_{h}, \tau_{h}\right)} \frac{4^{h q}}{\rho^{q}}\left|u-k_{h+1}\right|^{q}+\frac{4^{h}}{\rho^{2}}\left|u-k_{h+1}\right|^{2}+1 \mathrm{~d} z\right]^{\frac{n+p}{n} \frac{q}{p_{\star}}}\right. \\
& \left.\quad \times\left|A\left(k_{h+1}, \rho_{h}, \tau_{h}\right)\right|^{1-\frac{q}{p_{\star}}}\right)^{\frac{2 \alpha}{q}} \\
& \leq c M\left(\left[\int_{A\left(k_{h+1}, \rho_{h}, \tau_{h}\right)} \frac{4^{2 h}}{\rho^{2}}\left|u-k_{h+1}\right|^{2}+1 \mathrm{~d} z\right]^{\frac{n+p}{n} \frac{q}{p_{\star}}}\right. \\
& \left.\quad \times\left|A\left(k_{h+1}, \rho_{h}, \tau_{h}\right)\right|^{1-\frac{q}{p_{\star}}}\right)^{\frac{2 \alpha}{q}}
\end{aligned}
$$

We proceed similarly as in the proof of Theorem 1.2 and conclude

$$
\int_{A\left(k_{h+1}, \rho_{h}, \tau_{h}\right)}\left|u-k_{h+1}\right|^{2} \mathrm{~d} z \leq J_{h}
$$

and

$$
\left|A\left(k_{h+1}, \rho_{h}, \tau_{h}\right)\right| \leq \frac{4^{(h+2) 2}}{k^{2}} J_{h} \leq 4^{(h+2) 2} J_{h} .
$$

Thus we get

$$
J_{h+1} \leq c M \frac{4^{2 \alpha\left(1+\frac{q}{p_{\star}} \frac{p}{n}\right) h}}{k^{\frac{2 \alpha}{q} \frac{2}{p_{\star}}\left(p_{\star}-q\right)} \rho^{2 \frac{n+p}{n} \frac{2 \alpha}{p_{\star}}}} J_{h}^{\left(1+\frac{p}{n} \frac{q}{p_{\star}}\right) \frac{2 \alpha}{q}} .
$$

Now we use Lemma 2.3 with

$$
\gamma=\left(1+\frac{p}{n} \frac{q}{p_{\star}}\right) \frac{2 \alpha}{q}-1=\frac{2 \alpha}{n+2}+\frac{2 \alpha}{r}-\frac{2}{r} .
$$

Note that the assumptions for $r$ and $\alpha$ imply that $\gamma>0$ holds and if we choose

$$
k=c\left[1+\left(\int_{Q_{\rho}\left(z_{0}\right)}|u|^{2} \mathrm{~d} z\right) \rho^{-\frac{4 \alpha}{p_{\star}} \frac{n+p}{n} \frac{1}{\gamma}} M^{\frac{1}{\gamma}}\right]^{\frac{q}{2 \alpha} \frac{p_{\star}}{2} \frac{\gamma}{p_{\star}-q}},
$$

we obtain

$$
\sup _{Q_{\frac{\rho}{2}}\left(z_{0}\right)} u \leq k
$$

Since $-u$ is also minimizer to an integrand satisfying the same growth conditions, we conclude that $u$ is bounded. But in order to get the optimal exponents in the estimate we have to iterate again and use that $u$ is locally bounded. Therefore we define

$$
\tilde{J}_{h}:=\int_{A\left(k_{h}, \rho_{h}, \tau_{h}\right)}\left|u-k_{h}\right|^{r} \mathrm{~d} z
$$


and take the function $\psi_{h}$ and $\zeta_{h}$ as above. If we use Lemmas 2.1 and 3.2 and the fact that $u$ is locally bounded, we obtain

$$
\begin{aligned}
\tilde{J}_{h+1} \leq & \int_{A\left(k_{h+1}, \bar{\rho}_{h}, \bar{\tau}_{h}\right)}\left|\left(u-k_{h+1}\right) \psi_{h} \zeta_{h}\right|^{r} \mathrm{~d} z \\
\leq & \|u\|_{L^{\infty}\left(Q_{\rho}\left(z_{0}\right)\right)}^{r-p_{\star}} \int_{A\left(k_{h+1}, \bar{\rho}_{h}, \bar{\tau}_{h}\right)}\left|\left(u-k_{h+1}\right) \psi_{h} \zeta_{h}\right|^{p_{\star}} \mathrm{d} z \\
\leq & c\|u\|_{L^{\infty}\left(Q_{\rho}\left(z_{0}\right)\right)}^{r-p_{\star}}\left[\sup _{t_{0}-\bar{\tau}_{h}<t<t_{0}} \int_{B_{\bar{p}_{h}}\left(x_{0}\right)}\left|\left(u-k_{h+1}\right)_{+}(\cdot, t)\right|^{2} \mathrm{~d} x\right. \\
& \left.\left.+\int_{A\left(k_{h+1}, \bar{\rho}_{h}, \bar{\tau}_{h}\right)}|D u|^{p}+\frac{2^{h p}}{\rho^{p}}\left|u-k_{h+1}\right|^{p} \mathrm{~d} z\right]^{\frac{n+p}{n}}\left|u-k_{h+1}\right|^{2}+1 \mathrm{~d} z\right]^{\frac{n+p}{n}} \\
\leq & c\|u\|_{L^{\infty}\left(Q_{\rho}\left(z_{0}\right)\right)}^{r-p_{\star}}\left[\frac{2^{2 h}}{\rho^{2}} \int_{A\left(k_{h+1}, \bar{\rho}_{h}, \bar{\tau}_{h}\right)}\right]^{\frac{n+p}{n}} \\
\leq & \left.c\|u\|_{L^{\infty}\left(Q_{\rho}\left(z_{0}\right)\right)}^{r-p_{\star}}\left(\frac{4^{h}}{\rho^{2}}\right)^{\frac{2}{r}}\left|A\left(k_{h+1}, \rho_{h}, \tau_{h}\right)\right|^{1-\frac{2}{r}}\right]^{\frac{n+p}{n}} \\
& \times\left[\left(\int_{A\left(k_{h+1}, \rho_{h}, \tau_{h}\right)}^{\left.\left|u-k_{h+1}\right|^{r}+1 \mathrm{~d} z\right)^{(3.11)}} .\right.\right.
\end{aligned}
$$

Since $k \geq 1$, we have

$$
\int_{A\left(k_{h+1}, \rho_{h}, \tau_{k}\right)}\left|u-k_{h+1}\right|^{r} \mathrm{~d} z \leq \tilde{J}_{h}
$$

and

$$
\left|A\left(k_{h+1}, \rho_{h}, \tau_{h}\right)\right| \leq \frac{2^{(h+2) r}}{k^{r}} \tilde{J}_{h} \leq 2^{(h+2) r} \tilde{J}_{h} .
$$

With these estimates (3.11) turns into

$$
\tilde{J}_{h+1} \leq c\|u\|_{L^{\infty}\left(Q_{\rho}\left(z_{0}\right)\right)}^{r-p_{\star}} \frac{4^{h \frac{n+p}{n}}}{\rho^{2 \frac{n+p}{n}}} \frac{1}{k^{(r-2) \frac{n+p}{n}}} \tilde{J}_{h}^{\frac{n+p}{n}}
$$

and we can use Lemma 2.3 with

$$
\gamma=\frac{p}{n}, \quad M=\frac{c\|u\|_{L^{\infty}\left(Q_{\rho}\left(z_{0}\right)\right)}^{r-p_{\star}}}{\rho^{2 \frac{n+p}{n}} k^{(r-2) \frac{n+p}{n}}} \quad \text { and } \quad b=4^{\frac{n+p}{n}} .
$$

Now we choose

$$
k=c\|u\|_{L^{\infty}\left(Q_{\rho}\left(z_{0}\right)\right)}^{\frac{r-p_{\star}}{r-2} \frac{n}{n+p}}\left(1+\rho^{-\frac{2(n+p)}{p}} \int_{Q_{\rho}\left(z_{0}\right)}|u|^{r} \mathrm{~d} z\right)^{\frac{p}{(r-2)(n+p)}}
$$


for a constant $c$ depending on $n, p, q, \nu, L, r$ and get

$$
\int_{Q_{\rho, \rho^{2}}\left(z_{0}\right) \cap\{u>0\}}|u|^{r} \mathrm{~d} z=\tilde{J}_{0} \leq M^{-\frac{1}{\gamma}} b^{-\frac{1}{\gamma^{2}}} .
$$

But this implies

$$
\sup _{Q_{\frac{\rho}{2}}\left(z_{0}\right)} u \leq c\|u\|_{L^{\infty}\left(Q_{\rho}\left(z_{0}\right)\right)}^{\frac{r-p_{\star} \frac{n}{n+p}}{r-2}}\left(1+\rho^{-\frac{2(n+p)}{p}} \int_{Q_{\rho}\left(z_{0}\right)}|u|^{r} \mathrm{~d} z\right)^{\frac{p}{(r-2)(n+p)}},
$$

with a constant $c$ only depending on $n, p, q, \nu, L, r$. With the definition

$$
M_{h}:=\sup _{Q_{\rho_{h}, \tau_{h}}\left(z_{0}\right)} u
$$

we gain from (3.12)

$$
M_{h} \leq c\left(1+\rho^{-\frac{2(n+p)}{p}} \int_{Q_{\rho}\left(z_{0}\right)}|u|^{r} \mathrm{~d} z\right)^{\frac{p}{(r-2)(n+p)}} M_{h+1}^{\frac{r-p_{\star}}{r-2} \frac{n}{n+p}} .
$$

Since the assumption

$$
r>\frac{n(2-p)}{p}
$$

is equivalent to

$$
\frac{r-p_{\star}}{r-2} \frac{n}{n+p}<1
$$

we can use Lemma 2.4 with

$$
\alpha=1-\frac{r-p_{\star}}{r-2} \frac{n}{n+p}=\frac{\lambda_{r}}{(r-2)(n+p)}
$$

and

$$
C=\left(1+\rho^{-\frac{2(n+p)}{p}} \int_{Q_{\rho}\left(z_{0}\right)}|u|^{r} \mathrm{~d} z\right)^{\frac{p}{(r-2)(n+p)}}
$$

and achieve

$$
\sup _{Q_{\frac{\rho}{2}}\left(z_{0}\right)} u \leq c\left(1+\rho^{-\frac{2(n+p)}{p}} \int_{Q_{\rho}\left(z_{0}\right)}|u|^{r} \mathrm{~d} z\right)^{\frac{p}{\lambda_{r}}}
$$

where we used the abbreviation

$$
\lambda_{r}=n(p-2)+r p .
$$

Again we can conclude that $-u$ has the same upper bound and hence the proof is completed. 


\section{4. $L_{\mathrm{loc}}^{\infty}$-bound for anisotropic integrands}

Now we consider parabolic minimizers of integrands with anisotropic growth conditions. Our approach will be similar to the procedure of the last section. We will also start with a Caccioppoli inequality on superlevel sets.

Lemma 4.1. Let $u \in C\left([0, T] ; L^{2}(\Omega)\right) \cap L^{p}\left(0, T ; W^{1,\left(p_{i}\right)}(\Omega)\right)$ be a local minimizer, where the integrand satisfies (1.5) and $p_{i} \leq p_{\star}$ holds. Then for all cylinders $Q_{\rho, \rho^{2}}\left(z_{0}\right) \Subset \Omega_{T}$ and $k, \tau_{1}, \tau_{2}>0$ with $\frac{1}{2} \rho \leq \rho_{1}<\rho_{2} \leq \rho, t_{0}-\rho^{2} \leq$ $t_{0}-\tau_{2}<t_{0}-\tau_{1} \leq t_{0}-\frac{1}{2} \rho^{2}$ and $p_{i} \leq \delta \leq p_{\star}$ we have

$$
\begin{aligned}
& \sup _{t_{0}-\tau_{1}<t<t_{0}} \int_{B_{\rho_{1}}\left(x_{0}\right)}\left|(u-k)_{+}(\cdot, t)\right|^{2} \mathrm{~d} x+\int_{A\left(k, \rho_{1}, \tau_{1}\right)} \sum_{i=1}^{n}\left|D_{i} u\right|^{p_{i}} \mathrm{~d} z \\
& \leq c \int_{A\left(k, \rho_{2}, \tau_{2}\right)} \frac{\left|(u-k)_{+}\right|^{2}}{\tau_{2}-\tau_{1}}+\frac{\left|(u-k)_{+}\right|^{\delta}}{\left(\rho_{2}-\rho_{1}\right)^{\delta}}+1 \mathrm{~d} z
\end{aligned}
$$

with a constant $c=c\left(n, p_{i}, \nu, L\right)$.

Proof. For any $k>0$ we choose

$$
\varphi=-\chi_{\varepsilon} \psi^{p} \zeta^{p}(u-k)_{+}
$$

as testing function, where the functions $\psi \in C^{1}(\mathbb{R})$ and $\zeta \in C_{0}^{1}\left(B_{\rho}\right)$ are cut-off functions with $0 \leq \psi, \zeta \leq 1$. Additionally we can choose $\zeta$ such that $\zeta \equiv 1$ on $B_{\rho_{1}}\left(x_{0}\right), \zeta \equiv 0$ outside of $B_{\rho_{2}}\left(x_{0}\right)$ and $0 \leq|D \zeta| \leq 2 /\left(\rho_{2}-\rho_{1}\right)$ holds. For $\psi$ we can assume that $\psi \equiv 0$ on $\left(t_{0}-\rho^{2}, t_{0}-\tau_{2}\right), \psi \equiv 1$ on $\left(t_{0}-\tau_{1}, t_{0}\right)$ and $0 \leq \psi^{\prime} \leq 2 /\left(\tau_{2}-\tau_{1}\right)$ holds. Last, the function $\chi_{\varepsilon}$ is defined by

$$
\chi_{\varepsilon}:= \begin{cases}1 & t_{0}-\rho^{2} \leq t \leq \tau \\ 1-\frac{1}{\varepsilon}(t-\tau) & \tau<t<\tau+\varepsilon \\ 0 & \text { otherwise }\end{cases}
$$

for $\tau \in\left(t_{0}-\tau_{1}, t_{0}-\varepsilon\right)$ and $0<\varepsilon \ll 1$. We can assume that $\partial_{t} u \in L^{2}\left(\Omega_{T}\right)$ holds, otherwise, we use Steklov-averages to justify $\varphi$ as testing function in (1.6). We treat the term with the time derivative in the same way as in the proof of Lemma 3.2 and get

$$
\begin{aligned}
& \int_{\operatorname{spt} \varphi} u \cdot \partial_{t} \varphi \mathrm{d} z \\
& =-\int_{A\left(k, \rho_{2}, \tau_{2}\right)} \frac{1}{2}\left|(u-k)_{+}\right|^{2}\left(\chi_{\varepsilon}^{\prime} \psi^{p} \zeta^{p}+p \chi_{\varepsilon} \psi^{p-1} \psi^{\prime} \zeta^{p}\right) \mathrm{d} z \\
& \quad \stackrel{\varepsilon \downarrow 0}{\longrightarrow} \frac{1}{2} \int_{B_{\rho_{2}}\left(x_{0}\right)} \zeta^{p}\left|(u-k)_{+}(\cdot, \tau)\right|^{2} \mathrm{~d} x \\
& \quad-\frac{p}{2} \int_{A\left(k, \rho_{2}, \tau_{2}\right)}\left|(u-k)_{+}\right|^{2} \psi^{p-1} \psi^{\prime} \zeta^{p} \chi_{\left(t_{0}-\tau_{1}, \tau\right)} \\
& \geq \frac{1}{2} \int_{B_{\rho_{1}}\left(x_{0}\right)}\left|(u-k)_{+}(\cdot, \tau)\right|^{2} \mathrm{~d} x-p \int_{A\left(k, \rho_{2}, \tau_{2}\right)} \frac{\left|(u-k)_{+}\right|^{2}}{\tau_{2}-\tau_{1}} \mathrm{~d} z
\end{aligned}
$$


With the computation

$$
\left.D_{i} \varphi=-\psi^{p} \chi_{\varepsilon}\left(p \zeta^{p-1} D_{i} \zeta(u-k)_{+}\right)+\zeta^{p} D_{i} u \chi_{[u>k]}\right)
$$

and the growth assumption (1.5) we estimate

$$
\begin{aligned}
& \int_{A\left(k, \rho_{2}, \tau_{2}\right)} f(x, t, u+\varphi, D u+D \varphi) \mathrm{d} z \\
& \leq L \int_{A\left(k, \rho_{2}, \tau_{2}\right)} \sum_{i=1}^{n}\left|D_{i} u+D_{i} \varphi\right|^{p_{i}}+1 \mathrm{~d} z \leq c \int_{A\left(k, \rho_{2}, \tau_{2}\right)} \sum_{i=1}^{n} \\
& \quad \times\left[\left(\chi_{\varepsilon} \psi^{p} \zeta^{p-1}\right)^{p_{i}}\left|D_{i} \zeta\right|^{p_{i}}(u-k)_{+}^{p_{i}}+\left|D_{i} u\right|^{p_{i}}\left(1-\chi_{\varepsilon} \psi^{p} \zeta^{p}\right)^{p_{i}}\right]+1 \mathrm{~d} z \\
& \leq c \int_{A\left(k, \rho_{2}, \tau_{2}\right)} \sum_{i=1}^{n}\left[\left|D_{i} \zeta\right|^{p_{i}}(u-k)_{+}^{p_{i}}+\left|D_{i} u\right|^{p_{i}}\left(1-\chi_{\varepsilon} \psi^{p} \zeta^{p}\right)\right]+1 \mathrm{~d} z .
\end{aligned}
$$

Further we use the growth assumption (1.5)

$$
\int_{A\left(k, \rho_{2}, \tau_{2}\right)} f(x, t, u, D u) \mathrm{d} z \geq \nu \int_{A\left(k, \rho_{2}, \tau_{2}\right)} \sum_{i=1}^{n}\left|D_{i} u\right|^{p_{i}} \mathrm{~d} z
$$

and if we plug in our estimates and let $\varepsilon \downarrow 0$, we obtain for any $\tau \in\left(t_{0}-\tau_{1}, t_{0}\right)$

$$
\begin{aligned}
& \int_{B_{\rho_{1}}\left(x_{0}\right)}\left|(u-k)_{+}(\cdot, \tau)\right|^{2} \mathrm{~d} x+\int_{A\left(k, \rho_{2}, \tau_{2}\right)} \sum_{i=1}^{n}\left|D_{i} u\right|^{p_{i}} \mathrm{~d} z \\
& \leq c \int_{A\left(k, \rho_{2}, \tau_{2}\right)} \frac{\left|(u-k)_{+}\right|^{2}}{\tau_{2}-\tau_{1}}+1 \\
& \quad+\sum_{i=1}^{n}\left(\frac{\left|(u-k)_{+}\right|^{p_{i}}}{\left(\rho_{2}-\rho_{1}\right)^{p_{i}}}+\left|D_{i} u\right|^{p_{i}}\left(1-\psi^{p} \zeta^{p} \chi_{\left(t_{0}-\tau_{1}, \tau\right)}\right)\right) \mathrm{d} z .
\end{aligned}
$$

Now we add

$$
c \int_{A\left(k, \rho_{2}, \tau_{2}\right)} \sum_{i=1}^{n}\left|D_{i} u\right|^{p_{i}} \zeta^{p} \psi^{p} \chi_{\left(t_{0}-\tau_{1}, \tau\right)} \mathrm{d} z
$$

on both sides, divide the inequality by $c+1$ and obtain

$$
\begin{aligned}
& \frac{1}{c+1} \int_{B_{\rho_{1}}\left(x_{0}\right)}\left|(u-k)_{+}(\cdot, \tau)\right|^{2} \mathrm{~d} x+\int_{A\left(k, \rho_{1}, \tau_{1}\right)} \chi_{\left(t_{0}-\tau_{1}, \tau\right)} \sum_{i=1}^{n}\left|D_{i} u\right|^{p_{i}} \mathrm{~d} z \\
& \leq \frac{c}{c+1} \int_{A\left(k, \rho_{2}, \tau_{2}\right)} \sum_{i=1}^{n}\left|D_{i} u\right|^{p_{i}} \mathrm{~d} z \\
& \quad+\int_{A\left(k, \rho_{2}, \tau_{2}\right)} \sum_{i=1}^{n} \frac{\left|(u-k)_{+}\right|^{p_{i}}}{\left(\rho_{2}-\rho_{1}\right)^{p_{i}}}+\frac{\left|(u-k)_{+}\right|^{2}}{\tau_{2}-\tau_{1}}+1 \mathrm{~d} z .
\end{aligned}
$$


Now we take the supremum over $\tau \in\left(t_{0}-\tau_{1}\right)$ and get

$$
\begin{aligned}
& \frac{1}{c+1} \sup _{t_{0}-\tau_{1}<t<t_{0}} \int_{B_{\rho_{1}}\left(x_{0}\right)}\left|(u-k)_{+}(\cdot, t)\right|^{2} \mathrm{~d} x+\int_{A\left(k, \rho_{1}, \tau_{1}\right)} \sum_{i=1}^{n}\left|D_{i} u\right|^{p_{i}} \mathrm{~d} z \\
& \leq \frac{c}{c+1} \int_{A\left(k, \rho_{2}, \tau_{2}\right)} \sum_{i=1}^{n}\left|D_{i} u\right|^{p_{i}} \mathrm{~d} z \\
& \quad+\int_{A\left(k, \rho_{2}, \tau_{2}\right)} \sum_{i=1}^{n} \frac{\left|(u-k)_{+}\right|^{p_{i}}}{\left(\rho_{2}-\rho_{1}\right)^{p_{i}}}+\frac{\left|(u-k)_{+}\right|^{2}}{\tau_{2}-\tau_{1}}+1 \mathrm{~d} z .
\end{aligned}
$$

Then Lemma 2.5 and Young's inequality imply

$$
\begin{aligned}
& \sup _{\tau_{2}<t<t_{0}} \int_{B_{\rho_{1}}\left(x_{0}\right)}\left|(u-k)_{+}(\cdot, t)\right|^{2} \mathrm{~d} x+\int_{A\left(k, \rho_{1}, \tau_{2}\right)} \sum_{i=1}^{n}\left|D_{i} u\right|^{p_{i}} \mathrm{~d} z \\
& \leq c \int_{A\left(k, \rho_{2}, \tau_{1}\right)} \frac{\left|(u-k)_{+}\right|^{2}}{\tau_{2}-\tau_{1}}+\sum_{i=1}^{n} \frac{\left|(u-k)_{+}\right|^{p_{i}}}{\left(\rho_{2}-\rho_{1}\right)^{p_{i}}}+1 \mathrm{~d} z \\
& \leq c \int_{A\left(k, \rho_{2}, \tau_{1}\right)} \frac{\left|(u-k)_{+}\right|^{2}}{\tau_{2}-\tau_{1}}+\frac{\left|(u-k)_{+}\right|^{\delta}}{\left(\rho_{2}-\rho_{1}\right)^{\delta}}+1 \mathrm{~d} z,
\end{aligned}
$$

with $c=c\left(n, p_{i}, \nu, L\right)$.

Now we prove Theorem 1.7 for the case $p_{i}<p_{\star}$ for all $i \in\{1, \ldots, n\}$.

Proof of Theorem 1.7. We will use the notation from the proof of Theorem 1.2 and define the parameters just as in (3.4) and (3.5).

Now we choose $\psi_{h} \in C^{1}\left(\left(t_{0}-\rho^{2}, t_{0}\right)\right)$ and $\zeta_{h} \in C_{0}^{1}\left(B_{\rho}\left(x_{0}\right)\right)$ with $0 \leq \zeta_{h} \leq$ 1 and $\zeta_{h} \equiv 1$ in $B_{\rho_{h+1}}\left(x_{0}\right), \zeta_{h} \equiv 0$ outside of $B_{\bar{\rho}_{h}}\left(x_{0}\right)$ and $\left|D \zeta_{h}\right| \leq \frac{2^{h}}{\rho}$. Further we take $0 \leq \psi_{h} \leq 1$ with $\psi_{h} \equiv 1$ in $\left(t_{0}-\tau_{k+1}, t_{0}\right)$ and $\psi_{h} \equiv 0$ for $t \leq t_{0}-\bar{\tau}_{h}$. With these choices and Lemmas 2.2 and 4.1 we have

$$
\begin{aligned}
J_{h+1} \leq & \int_{A\left(k_{h+1}, \bar{\rho}_{h}, \bar{\tau}_{h}\right)}\left|\left(u-k_{h+1}\right) \psi_{h} \zeta_{h}\right|^{\delta} \mathrm{d} z \\
\leq & \left(\int_{A\left(k_{h+1}, \bar{\rho}_{h}, \bar{\tau}_{h}\right)}\left|\left(u-k_{h+1}\right) \psi_{h} \zeta_{h}\right|^{p_{\star}} \mathrm{d} z\right)^{\frac{\delta}{p_{\star}}} \cdot\left|A\left(k_{h+1}, \bar{\rho}_{h}, \bar{\tau}_{h}\right)\right|^{1-\frac{\delta}{p_{\star}}} \\
\leq & c\left[\sup _{t_{0}-\bar{\tau}_{h}<t<t_{0}} \int_{B_{\bar{\rho}_{h}}\left(x_{0}\right)}\left|\left(u-k_{h+1}\right)_{+}\right|^{2}(\cdot, t) \mathrm{d} x+\int_{A\left(k_{h+1}, \bar{\rho}_{h}, \bar{\tau}_{h}\right)} \sum_{i=1}^{n}\left|D_{i} u\right|^{p_{i}} \mathrm{~d} z\right. \\
& \left.+\int_{A\left(k_{h+1}, \bar{\rho}_{h}, \bar{\tau}_{h}\right)} \sum_{i=1}^{n} \frac{2^{h p_{i}}}{\rho^{p_{i}}}\left|u-k_{h+1}\right|^{p_{i}} \mathrm{~d} z\right]^{\frac{n+p}{n} \frac{\delta}{p_{\star}}} \cdot\left|A\left(k_{h+1}, \rho_{h}, \tau_{h}\right)\right|^{1-\frac{\delta}{p_{\star}}}
\end{aligned}
$$




$$
\begin{aligned}
\leq & c\left[\int_{A\left(k_{h+1}, \rho_{h}, \tau_{h}\right)} \frac{2^{h \delta}}{\rho^{\delta}}\left|u-k_{h+1}\right|^{\delta}+\frac{4^{h}}{\rho^{2}}\left|u-k_{h+1}\right|^{2}+1 \mathrm{~d} z\right]^{\frac{n+p}{n} \frac{\delta}{p_{\star}}} \\
& \times\left|A\left(k_{h+1}, \rho_{h}, \tau_{h}\right)\right|^{1-\frac{\delta}{p_{\star}}} \\
\leq & c\left[\frac{4^{h \delta}}{\rho^{\delta}} \int_{A\left(k_{h+1}, \rho_{h}, \tau_{h}\right)}\left|u-k_{h+1}\right|^{\delta}+1 \mathrm{~d} z\right]^{\frac{n+p}{n} \frac{\delta}{p_{\star}}} \cdot\left|A\left(k_{h+1}, \rho_{h}, \tau_{h}\right)\right|^{1-\frac{\delta}{p_{\star}}}
\end{aligned}
$$

Now we can iterate this inequality in exactly the same way as in the proof of Theorem 1.2 and obtain the claim.

Theorem 1.8 can be proved by combining the methods of the proofs of Theorems 1.4 and 1.7 .

\section{Boundedness of solutions in the limit case}

To complete the proofs of the Theorems $1.2,1.4,1.7$ and 1.8 we have to consider the limit case $q=p_{\star}$ or respectively $p_{i}=p_{\star}$ for at least one $i \in\{1, \ldots, n\}$. We will start with variational solutions of integrands satisfying the $p, q$-growth condition

$$
\frac{2 n}{n+2}<p<q=p \frac{n+2}{n} .
$$

First we use Lemma 3.2 with $\delta=q$, hence we have for all $Q_{\rho, \rho^{2}}\left(z_{0}\right) \Subset \Omega_{T}$ and $k, \tau_{1}, \tau_{2}>0$ with $\frac{1}{2} \rho \leq \rho_{1}<\rho_{2} \leq \rho, t_{0}-\rho^{2} \leq t_{0}-\tau_{2}<t_{0}-\tau_{1} \leq t_{0}-\frac{1}{2} \rho^{2}$

$$
\begin{aligned}
& \sup _{t_{0}-\tau_{1}<t<t_{0}} \int_{B_{\rho_{1}}\left(x_{0}\right)}\left|(u-k)_{+}(\cdot, t)\right|^{2} \mathrm{~d} x+\int_{A\left(k, \rho_{1}, \tau_{1}\right)}|D u|^{p} \mathrm{~d} z \\
& \leq c \int_{A\left(k, \rho_{2}, \tau_{2}\right)} \frac{\left|(u-k)_{+}\right|^{2}}{\tau_{2}-\tau_{1}}+\frac{\left|(u-k)_{+}\right|^{q}}{\left(\rho_{2}-\rho_{1}\right)^{q}}+1 \mathrm{~d} z .
\end{aligned}
$$

Now we define $\rho_{h}, \bar{\rho}_{h}, \tau_{h}$ and $\bar{\tau}_{h}$ as in (3.4). But in contrast to Sect. 3 we set

$$
k_{h}:=k\left(1-\frac{1}{2^{h+1}}\right)
$$

and

$$
J_{h}:=\int_{A\left(k_{h}, \rho_{h}, \tau_{h}\right)}\left|u-k_{h}\right|^{q} \mathrm{~d} z .
$$

If we use (5.1) and Lemma 2.1 we can find an analogous estimate to (3.9)

$$
J_{h+1} \leq c 4^{h q \frac{n+p}{n}} \rho^{-q \frac{n+p}{n}} J_{h}^{\frac{n+p}{n}} .
$$

The difference between this estimate and (3.9) is, that we only have a $k$ dependence in the terms of $J_{h}$. But we also want to iterate this inequality with the help of Lemma 2.3. Therefore we need

$$
J_{0} \leq M^{-\frac{1}{\gamma}} b^{-\frac{1}{\gamma^{2}}},
$$


with $M=c \rho^{-q \frac{n+p}{n}}, b=2^{q \frac{n+p}{n}}$ and $\gamma=\frac{p}{n}$. But by the definition of

$$
J_{0}=\int_{Q_{\rho, \rho^{2}}\left(z_{0}\right) \cap\left\{u>\frac{1}{2} k\right\}}\left|u-\frac{1}{2} k\right|^{q} \mathrm{~d} z,
$$

we can choose $k>0$ big enough, such that (5.2) holds. Hence Lemma 2.3 implies

$$
\sup _{Q_{\frac{\rho}{2}}\left(z_{0}\right)} u \leq k
$$

i.e. $u$ is locally bounded. This approach can also be applied in the sub-critical case for anisotropic integrands.

\section{Acknowledgements}

Open access funding provided by [Paris Lodron University of Salzburg]. This work was partially supported by the Grant DFG-Project BO3598/1-1 "Evolutionsgleichungen mit $p, q$-Wachstum". Moreover, I want to express my gratitude to the Studienstiftung des deutschen Volkes.

Open Access. This article is distributed under the terms of the Creative Commons Attribution 4.0 International License (http://creativecommons.org/licenses/ by/4.0/), which permits unrestricted use, distribution, and reproduction in any medium, provided you give appropriate credit to the original author(s) and the source, provide a link to the Creative Commons license, and indicate if changes were made.

\section{References}

[1] Bögelein, V., Duzaar, F., Marcellini, P.: Existence of evolutionary variational solutions via the calculus of variations. J. Differ. Equ. 256(12), 3912-3942 (2014)

[2] Bögelein, V., Duzaar, F., Marcellini, P.: A time dependent variational approach to image restoration. SIAM J. Imaging Sci. 8(2), 968-1006 (2015)

[3] Cupini, G., Marcellini, P., Mascolo, E.: Regularity under sharp anisotropic general growth conditions. Discrete Contin. Dyn. Syst. Ser. B 11(1), 66-86 (2009)

[4] Cupini, G., Marcellini, P., Mascolo, E.: Local boundedness of solutions to quasilinear elliptic systems. Manuscripta Math. 137(3-4), 287-315 (2012)

[5] Cupini, G., Marcellini, P., Mascolo, E.: Local boundedness of minimizers with limit growth conditions. J. Optim. Theory Appl. 166(1), 122 (2015)

[6] DiBenedetto, E.: Degenerate Parabolic Equations. Springer, New York (1993)

[7] Föglein, A.: Regularity results for minimizers of integrals with $(2, q)$-growth in the Heisenberg group. PhD thesis, Friedrich-Alexander-Universität, ErlangenNürnberg (2009) 
[8] Fusco, N., Sbordone, C.: Local boundedness of minimizers in a limit case. Manuscripta Math. 69, 19-25 (1990)

[9] Giaquinta, M., Giusti, E.: On the regularity of the minima of variational integrals. Acta Mathematica 148, 31-46 (1982)

[10] Kinnunen, J., Lindqvist, P.: Pointwise behaviour of semicontinuous supersolutions to a quasilinear parabolic equation. Ann. Mat. Pura Appl. (4) 185(3), 411-435 (2006)

[11] Ladyzhenskaya, O.A., Uraltseva, N.N.: Linear and quasilinear elliptic equations. Academic Press, New York (1968)

[12] Lichnewsky, A., Temam, R.: Pseudosolutions of the time-dependent minimal surface problem. J. Differ. Equ. 30(3), 340-364 (1978)

[13] Marcellini, P.: Un exemple de solution discontinue dun probleme variationnel dans le cas scalaire. Istituto Matematico "U. Dini", n. 11, Universita di Firenze (1987)

[14] Marcellini, P.: Regularity of minimizers of integrals of the calculus of variations with non standart growth conditions. Arch. Mat. Mech. Anal. 105, 267$284(1989)$

[15] Mingqi, Y., Xiting, L.: Boundedness of solutions of parabolic equations with anisotropic growth conditions. Can. J. Math. 49(4), 798-809 (1997)

[16] Moscariello, G., Nania, L.: Hölder continuity of minimizers of functional with non standart growth conditions. Ricerce Mat. 40(2), 259-273 (1991)

[17] Wieser, W.: Parabolic Q-minima and minimal solutions to variational flow. Manuscripta Math. 59(1), 63-107 (1987)

Thomas Singer

Fachbereich Mathematik

Universität Salzburg

Hellbrunner Strasse 34

5020 Salzburg

Austria

e-mail: thomas.singer@sbg.ac.at

Received: 26 June 2015.

Accepted: 30 December 2015. 